\title{
Clinical Diagnosis of Temporomandibular Joint Arthritis: A Difficult Task
}

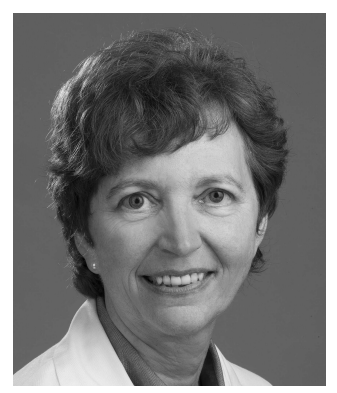

Juvenile idiopathic arthritis (JIA) frequently involves the temporomandibular joint (TMJ). TMJ involvement was already mentioned as a typical manifestation in the original publication by Sir Frederic Still ${ }^{1}$. Depending on the methods used, a rate of up to $93 \%$ of JIA patients with early inflammatory signs on TMJ imaging have been reported $2,3,4,5$. The rates reported for TMJ deformation and important mandibular growth disturbance are only slightly lower with $41-78 \%{ }^{6,7,8,9,10}$. Thus, early diagnosis and timely treatment of TMJ arthritis is important in efforts to prevent such irreversible damage.

A number of factors contribute to the difficulties connected to the diagnosis of TMJ arthritis:

Severity of consequences. The TMJ is a rather small joint and therefore may be regarded of minor importance. However, it has some unique features: movements in the TMJ are of high complexity, and the mechanical load during chewing and biting is higher than in any other joint of the body. Another peculiarity is that the growth of the mandible during childhood originates from a thin layer of growth cartilage cells located just beneath the joint surface ${ }^{11}$. Therefore, TMJ arthritis in children can not only lead to deformity and destruction of the mandibular head but can also severely affect the growth and development of the whole mandible. Especially in cases with early onset of TMJ arthritis, the resulting growth failure of the mandible will lead to hypognathism and retrognathism and may have important esthetic and functional consequences, the most severe form being a mandibular malformation also called "bird's face."

Lack of early symptoms and clinical signs. In the majority of patients with JIA, TMJ arthritis seems to be asymptomatic. Pain is very unreliable because it is reported in only $14-20 \%$ of patients with manifest imaging signs of TMJ arthritis ${ }^{3,8}$. Older children may be better able to report pain than young children $^{7}$, and certain manifestations of pain, such as pain on chewing and yawning, are better predictors of TMJ arthritis than others.
Many studies have found a significant correlation between TMJ arthritis and functional symptoms such as restricted mouth opening, impaired translational jaw movement, noises on TMJ movement, and asymmetric jaw movements $3,7,8,12$. However, clinical signs with high reliability are related to manifest condylar growth failure in 1 or both condyles, i.e., when irreversible damage has already occurred ${ }^{4}$. Another problem encountered in clinical examination is the fact that a TMJ can never be examined independently. The $2 \mathrm{TMJ}$ can move together only because of the bridging connection between them formed by the mandible, a unique situation in our body. This situation certainly adds to the difficulties encountered with the clinical evaluation of the TMJ.

A variety of imaging methods. Imaging methods used to assess the outcome of TMJ arthritis include conventional radiographs (lateral cephalogram, orthopantomogram), computerized tomogram (CT), cone beam $\mathrm{CT}$, magnetic resonance image (MRI), and ultrasound (US). Most data, and especially data on the longterm course of changes associated with TMJ arthritis, are available for conventional radiographs and CT. These imaging modalities are very well suited to show the bony situation of the TMJ and the mandible and are therefore always needed for the planning and documentation of orthodontic treatments. However, neither method is able to show changes in the soft tissue such as synovial thickening, joint effusions, or cartilage changes, and they are therefore not suitable for the detection of early inflammation before bony changes have occurred.

US, in contrast, is a method very well suited for examination of soft tissue and fluid collections. Further, US is widely available, relatively cheap, and easy to perform even in young children. Unfortunately, US has not lived up to these high expectations; it failed to correctly detect early TMJ arthritis in several studies from different centers ${ }^{3,4}$.

MRI, in contrast, can detect early inflammatory changes reliably even before structural changes have occurred and is

See Clinical symptoms in diagnosing JIA-TMJ, page 1871 
increasingly used in children with JIA as a screening method for the early diagnosis of TMJ arthritis ${ }^{3,4,5}$. Limitations for the use of MRI are its relatively high cost, limited availability, and long examination time, necessitating sedation for younger children. In addition, in older children the high prevalence of fixed dental appliances may further limit the use of MRI for the diagnosis and followup of TMJ arthritis.

In this issue of The Journal, Koos, et al present results of their study about development of a clinical score for diagnosis of TMJ arthritis in patients with JIA ${ }^{13}$. The score is based on 5 well-defined clinical items, which were tested as single items as well as in all possible combinations. Interestingly, the clinical findings are compared not only to MRI findings of the same patients performed within 1 month from clinical examination but also to clinical findings in a matched control cohort of healthy children.

Before discussing their results, however, let us ask ourselves what the requirements would be for a useful clinical score. Of course the ideal result would be the detection of an examination method that is easy to perform and allows a reliable diagnosis of TMJ arthritis, even in the population most difficult to examine, i.e., young children with limited collaboration capacity. Such a method could replace MRI for the diagnosis of TMJ arthritis. However, given the results of previous studies, it is highly unlikely that a single examination item will fulfill such a task. But there is still the hope that the right combination of items may turn out to have a far better performance than each single item.

For the less optimistic among us, a method with good reliability to indicate absence of inflammation in the TMJ would already be very helpful, because it would allow us to preselect patients for MRI examination. Based on current knowledge, the chances to find a single item with either the first or the second property is highly unlikely. Therefore, the idea of a score is very appealing.

Unfortunately, despite the remarkably large size of the cohort and the elaborate study design, the authors' conclusion is that the score is not sufficiently reliable to predict the presence or absence of active TMJ arthritis in children with JIA. Interestingly, the least reliable item in this study was mouth opening capacity. This is a surprise because most studies so far have found a strong association between restricted mouth opening capacity with TMJ involvement in patients with JIA. However, most of those studies were done in patients with longstanding JIA. Further, the diagnosis of TMJ involvement in most studies was based on radiographic signs; that is, bony changes were already present in these patients and may have contributed to the functional loss. On the other hand, as Koos, et al correctly point out, the wide range of normal values for mouth opening capacity among healthy children already foreshadows the study result that a single measurement of a single patient is unlikely to be a reliable predictor of TMJ pathology ${ }^{14}$.
When combining different clinical items, Koos, et al found that every additional item improved the predictive value of the score. However, none of the items tested nor combination of items was found to stand out. Unfortunately, the items did not perform any better for the negative prediction, i.e., they were also unable to reliably exclude active TMJ arthritis.

Their study once more underscores the difficulties connected to the early clinical diagnosis of TMJ arthritis. Given the severe consequences of a missed diagnosis and delayed treatment of TMJ arthritis, MRI of the TMJ must be recommended, at least in children with early onset JIA (because the effect size of mandibular growth failure is larger in younger children), and in children without systemic disease-modifying treatment of their JIA. Meanwhile, the search for an easier diagnosis method must go on.

ROTRAUD SAURENMANN, MD,

Director, Department of Pediatrics, Cantonal Hospital,

Brauerstr. 15

CH-8400 Winterthur, Switzerland

Address correspondence to Dr. Saurenmann;

E-mail: traudel.saurenmann@ksw.ch

\section{REFERENCES}

1. Still GF. On a form of chronic joint disease in children. Med Chir Trans 1897;80:47-60.9

2. Kuseler A, Pedersen TK, Gelineck J, Herlin T. A 2 year followup study of enhanced magnetic resonance imaging and clinical examination of the temporomandibular joint in children with juvenile idiopathic arthritis. J Rheumatol 2005;32:162-9.

3. Weiss PF, Arabshahi B, Johnson A, Bilaniuk LT, Zarnow D, Cahill AM, et al. High prevalence of temporomandibular joint arthritis at disease onset in children with juvenile idiopathic arthritis, as detected by magnetic resonance imaging but not by ultrasound. Arthritis Rheum 2008;58:1189-96.

4. Muller L, Kellenberger CJ, Cannizzaro E, Ettlin D, Schraner T, Bolt IB, et al. Early diagnosis of temporomandibular joint involvement in juvenile idiopathic arthritis: a pilot study comparing clinical examination and ultrasound to magnetic resonance imaging. Rheumatology 2009;48:680-5.

5. Saurenmann RK, Hauser R, Schroeder S, Cannizzaro E, Muller L, Kellenberger CJ. How important is early magnetic resonance imaging of the temporomandibular joint for the treatment of children with juvenile idiopathic arthritis? [abstract]. Arthritis Rheum 2011;63 Suppl:S106.

6. Pedersen TK, Jensen JJ, Melsen B, Herlin T. Resorption of the temporomandibular condylar bone according to subtypes of juvenile chronic arthritis. J Rheumatol 2001;28:2109-15.

7. Olson L, Eckerdal O, Hallonsten AL, Helkimo M, Koch G, Gare BA. Craniomandibular function in juvenile chronic arthritis. A clinical and radiographic study. Swed Dent J 1991;15:71-83.

8. Twilt M, Mobers SM, Arends LR, ten Cate R, van Suijlekom-Smit L. Temporomandibular involvement in juvenile idiopathic arthritis J Rheumatol 2004;31:1418-22.

9. Billiau AD, Hu Y, Verdonck A, Carels C, Wouters C. Temporomandibular joint arthritis in juvenile idiopathic arthritis: prevalence, clinical and radiological signs, and relation to dentofacial morphology. J Rheumatol 2007;34:1925-33.

10. Cannizzaro E, Schroeder S, Muller LM, Kellenberger CJ,

Personal non-commercial use only. The Journal of Rheumatology Copyright @ 2014 . All rights reserved. 
Saurenmann RK. Temporomandibular joint involvement in children with juvenile idiopathic arthritis. J Rheumatol 2011;38:510-5.

11. Copray JC, Dibbets JM, Kantomaa T. The role of condylar cartilage in the development of the temporomandibular joint. Angle Orthod 1988;58:369-80.

12. Pedersen TK, Kuseler A, Gelineck J, Herlin T. A prospective study of magnetic resonance and radiographic imaging in relation to symptoms and clinical findings of the temporomandibular joint in children with juvenile idiopathic arthritis. J Rheumatol 2008;35:1668-75.
13. Koos B, Twillt M, Kyank U, Fischer-Brandies H, Gassling V, Tzaribachev N. Reliability of clinical symptoms in diagnosing temporomandibular joint arthritis in juvenile idiopathic arthritis. J Rheumatol 2014;41:1871-7.

14. Müller L, van Waes H, Langerweger C, Molinari L, Saurenmann RK. Maximal mouth opening capacity: percentiles for healthy children 4-17 years of age. Pediatr Rheumatol Online J 2013;11:17.

J Rheumatol 2014;41:1734-6; doi:10.3899/jrheum.140851 